\title{
Preconception Sex Selection in Traditional Persian Medicine: A Review of the Literature
}

\author{
Seyde Sedighe Yousefi ${ }^{1,2,3}{ }^{\circledR}$, Roshanak Mokaberi Nejad ${ }^{4}$, Mojgan Tansaz ${ }^{4}$, Soodabeh Bioos ${ }^{5}$, Zeinab \\ Hamzehgardeshi ${ }^{1,6^{*}}$ (D)
}

\begin{abstract}
Objectives: This review investigates the existing research with regard to a variety of different suggested methods by traditional Persian medicine (TPM) about the baby's sex selection.

Materials and Methods: A comprehensive scoping review including five stages was conducted to summarize evidence on preimplantation sex selection in TPM. The research question and relevant studies were identified in stages 1 and 2, respectively. Then, studies were selected and data were charted in stages 3 and 4, and finally, the results were collated, summarized, and reported in stage 5.

Results: Accordingly, 7 general categories were arranged, including the temperament of the sexual organs in a man, the temperament of sexual organs in a woman, the type of male semen, and timing and manner of sexual intercourse for fetus sex induction purposes. Other categories were the effect of environmental factors, the couple's psychic states during the course of love-making, the couple's age, and measures for male sex induction.

Conclusions: This review summarizes the existing research regarding different suggested methods by TPM on the baby's sex selection. These could well be the basis for further prospective scientific research and investigation.

Keywords: Sex selection, Traditional medicine, Male fetus
\end{abstract}

\section{Introduction}

The history of the determination of one's child sex before conception itself has its roots in previous centuries. Various cultural, social, religious, economic, and medical nature (e.g., some X chromosome dependent diseases) causes together persuade couples to opt for either traditional or modern procedures to select their offspring sex $(1,2)$. Striking familial balance is itself among other parameters affecting child sex selection (3).

Thus, determining the secondary sexual ratio is applicable in the matrix of society as a hygienic index. This actually consists of viable fetuses' sex and makes out around 106 males to 100 females (4). Overall, males are all but equal to females within human societies. However, societies of different races have male to female ratios of varying numerical values due to genetic differences. For instance, the blacks have more female children as compared with the whites (5). It is also true that psychic conditions such as stress arising from one's life stringent conditions cause changes in sexual activities, leading to changes in the mentioned sexual ratio (6).

Various laboratory methods are now using for selecting babies' sex before conception, including pre-implantation genetic diagnosis (7), Microsort (7, 3, 8), albumin gradient method $(3,9)$, and simple modified swim-up $(3,10)$.

In view of the cited methods, it could be opined that high costs of sex induction on the one hand, and their invasiveness, on the other hand, have led to limitations to parents so much so that parents have always been searching for less dangerous, more certain, and economical methods. Therefore, nowadays, research further focuses on acceptable, less costly, and less invasive methods.

The Iranian school of medicine, with its multi-millennia history, is a resourceful fountainhead of findings and experiences on the part of great medical sages such as Avicenna, Rhazes, and the like. Thus, it seems necessary to test such findings and experiences.

Traditional Persian medicine (TPM) sources and literature have well delved into the methods of baby sex selection. Given that TPM ages consider nutrition as the most important parameter in treatment, prevention, and general health management, they have not naturally hesitated to put most emphasis on baby's sex induction on the exact dietary method. 
The current scoping review summaries the existing research on various suggested methods by TPM regarding a baby's sex selection. These could well be the basis for further prospective scientific research and investigation in this respect.

\section{Materials and Methods}

Sample Selection and Characteristics

This comprehensive scoping review was performed to summarize evidence on pre-implantation sex selection in TPM.

Five stages were used for conducting a scoping study, including identifying the research question and relevant studies, selecting the studies, charting the data, and collating, summarizing, and reporting the results (11).

This is a review of studies over valid TPM literature, having their roots in various previous centuries, including the Canon of Medicine (by Avicenna), Exir-e-Azam, Fosool Aboghrat, Tadbir Hobala va Al-Morzae, Kholase AlHekma, Mojarrabat Farangi, Al-Aghraz Al-Tebbia va AlMabahes Al-Alaia, and some other books. First, materials were noted regarding the topic of "Fi Tadbir Al-Azkar" on "how to turn the original fetus into a son". Then, they were summarized whereas repetitive or infeasible measures were all excluded from further investigation. Then, the classified materials were included in the current review. The TM resources were selected based on the review question, and the hand search was used for this review.

\section{Inclusion Criteria}

Resources describing the methods of sex selection in TM were included in this review.

\section{Exclusion Criteria}

On the other hand, traditional resources which did not explain sex selection methods were excluded from the investigation.

\section{Results}

Factors Affecting the Procreation of a Male/Female Fetus in PM

Various parameters play an effective role in determining the fetus sex, including:

- The temperament of the sexual organs in a man;

- The temperament of the sexual organs in a woman;

- The type of male/female semen;

- Timing and method of sexual intercourse;

- Environmental factors;

- Psychic state of the couple during the course of sexual action;

- $\quad$ The age of the couple (12-15).

Given that the temperament of male/female sexual organs is one of the most important factors in the fetus sex induction, this section embarks on a summary investigation into the temperaments of each of the two organs.
The Temperament of Testis in a Man

The temperament of the testes is a highly effective parameter in sperm production by a man. It is noteworthy that the procreation of a male/female fetus (i.e., the induction of male/female sex) can increase or decrease depending on testes' temperament (3).

\section{Symptomatology}

There is a higher likelihood of male fetus induction as for those fathers whose testis have the warm temperament. Some of the distinctive signs of such warm temperament are as follows:

- Predominance of sexual desire;

- Higher speed of hair growth;

- Higher speed of pubic hair growth;

- More thickness of pubic hair;

- More viscous semen;

- Higher frequency of nocturnal wetting;

- Greater volume of semen;

- Some level of physiological harm in case of discarding sexual intercourse.

When the temperament turns out to be cold, either no fetuses are conceived or there is a higher probability of being female in the case of conception. The most prominent features of such cold nature are as follows:

- Lack and/or shortage of sexual desire;

- Softness of pubic hair;

- Less density of pubic hair;

- Less viscous semen;

- Latency of puberty;

- Slow ejaculation rate;

- Lower, colder volumes of semen.

Such people will not be extremely hurt if they discard sexual intercourse. On the other hand, the temperament of female gonads is also of significance in inducting the fetus's sex (13).

The Type of Male Semen

Other significant parameters in determining the fetus's gender are female/male semen strength, semen weakness, semen warmth or coldness, and semen rarity. The more the semen should be of a warm temperament, namely, the right testis and/or the right gonad (due to proximity to the liver and less humidity), the higher would be the probability of male gender induction in case of the semen is placed into the right side of the uterus. Various opinions have been expressed over this issue, some of which are as follows:

- When both male and female semen are "strong", the gender induction will be perfectly male.

- When male semen is strong while female semen is weak, the following two cases might occur:

A. When both male and female semen come together in equal proportions, there is a higher likelihood of bearing a son with slightly girlish characteristics. 
B. When male semen is quantitatively more than female semen, a son will be born of the highest level of perfection and probity.

- When female semen is quantitatively more than male semen, a girlish son will be born.

- When both male and female semen are feebly week, a girl will be born.

- When the female semen is feeble while the male semen is quantitatively more, a boyish girl of good strength will be born.

- When the female semen is weak whereas the female semen is quantitatively more than male semen, a girl of a weak build will be born.

In general, if the semen comes from the right testis or the right gonad, the fetus shall be male while the fetus shall be female if the semen comes from the left testis or the left gonad. In case the fetus is from the left side and turns out to be male, he will turn out to have characteristics similar to girls because of weakness, feebleness, the coolness of temperament, and the shortage of warmth. In case the semen comes from the right side of the male partner, going to the right side of the receiving female partner, then, the fetus will be a son.

- If the semen goes from the left side of the male partner to the right side of the receiving female partner, then, a boyish girl shall be born.

- A girlish boy shall be born in case the semen goes from the right side of the male partner to the left side of the receiving female partner $(13,14,16,17)$.

- Hippocrates and Aristotle mentioned that the fetus shall be a son if the man's semen starts its motion from his right side and finds its placement to the right side of the uterus $(13,18)$.

Dense, warm, dry semen would procreate male fetuses whereas cold, wet, and rare semen would procreate a female fetus (19). The male gender induction will be "accepted" if the woman's semen is extremely strong and warm. The opinion asserting that woman's semen should be weak for procreating male fetuses is simply wrong (13).

Avicenna has vividly declared in his Shefa that male sex shall not be made of the mother, and that matured, ripe semen from a strong mature man would give procreation to male fetuses. In other words, the cause of the fetus turning out to be male has been mentioned as male semen's warm temperament (by Avicenna). Later on, he mentions that for the fetus to turn out to be male, it would not be necessary for the man's semen to be highly warm. More precisely, it would be simple enough for the male semen to be of temperate temperament.

Then, for the fetus to turn out to be male, the natural cause would be the potential extant in the male partner's semen. No daughter will be born when the mentioned semen comes to be extremely warm (20).

For making the male semen stronger than that of the female, sexual intercourse should not be carried out for some time $(13,17)$. It is only later on that the male partner may well turn his attention to the semen. Nonetheless, the treatment should be continued if it is still rare. In case, it has turned out to be dense, again, the aforementioned treatment is observed for a couple of days to such an extent that the semen becomes even stronger. There have been some sages who believed that a son would be born only through the most dense semen (13).

Timing and Manner of Sexual Intercourse for the Fetus Sex Induction Purposes

1. The timing of sexual intercourse is one of the important parameters in sex determination. There has been a relatively wide spectrum of pieces of advice over this specific issue. In this regard, some sages asserted that there would be a higher likelihood of the fetus' turning out to be a son if the partners perform the sexual intercourse on the day of religious ritual ablution (Ghosl), in Persian that is to say, on the day when clearing from menstruation takes place. In case the sexual intercourse takes place on the second and third days after Taharat (clearing from menstruation), sex induction would be in favor of a daughter and a son, respectively. On the fourth day, it would make a daughter while on the fifth day, the likelihood increases for a son. From the fifth until the twelfth day, there is a high likelihood for male sex induction on odd days (16). There are other sages, however, indicated that sexual intercourse on the day of religious ritual ablution (Ghosl) up to the eighth day gives rise to a higher probability of male sex induction.

2. Nevertheless, another recommendation is for the couple to not have any sexual intercourse for some time. Such a period of no-sex, nonetheless, should not be lengthened for the simple reason that it could well bring about semen deterioration.

3. It would be better for male sex induction to have the female partner leaning to the right during the sexual intercourse in such a manner as to make it possible for the male semen to flow into the right side of the uterus.

4. The male partner should delicately arouse his wife sexually before the very act of intercourse. This should be done with light heart and exhilarating mirth. In other words, sexual intercourse should not be initiated up until the husband finds himself truly aroused as well.

5. After the accomplishment of sexual intercourse, down to the time cervical contractions are turning relaxed, the husband should in no way separate from the wife. The wife, in her own turn, should not move at all $(12-14,17)$.

\section{The Effect of Environmental Factors}

Among other significant parameters in sex determination are environmental effects $(12,14,21)$. In his book (Shefa), 
Avicenna considers the season, the living place, the climatic conditions, and the blowing of the winds as important factors in fetus sex induction. He further indicates that in case sexual intercourse occurs throughout North winds and inside North-bound domicile, there is a high likelihood for the fetus to be male (20). All during the blowing of South winds, in case it is warm during the course of summer, there is a high likelihood for the fetus to be female. Sexual intercourse right after getting "cleansed" from monthly menstruation --if taking place in cold towns during the course of winter on those specific days when North winds are blowing-- leads to male sex induction. This is due to the existence of warmth all during the day, which causes the semen to become warmer (16). It should also be taken into consideration that the human body tends to become cold and dry when winds come from the North or Northern areas (22).

Seasons could also be of significance in sex determination. For instance, springtime could vivify sexual libido according to its effect of suitability with the soul's temperament and strengthening of the faculty. This could well be the factor for increasing the level of fecundity amongst animals per se. It is at such time that the Mother of Nature tends more toward procreating male fetuses. When it becomes winter, the likelihood for the procreation of girl babies actually increases because of the predominance of cold over the human body and decreases in movements which lead to the annihilation of moisture (23).

The Couple's Psychic States During the Course of Lovemaking

Among other highly effective causes in determining the fetus' sex would be the psychic/mental state of couples during sexual intercourse. It has been advised that a man and a woman coming together for sex ought to arouse in one another peace of mind and exhilaration of mirthful nature. They should further pay attention to the fact that their mannerism stream of thoughts during sexual intercourse is also significant in sex induction so much so that thinking of strong men could well give rise to the fetus turning into a male one $(13,14,21,23)$.

\section{The Couple's Age}

During the course of the young age of couples, there is a higher likelihood of male sex induction while there is a higher likelihood for the fetus to turn out to be a female during older years of couples coming together $(13,14)$.

\section{Measures for Male Sex Induction}

Having known the general causes of sex induction, we can start delving into effective measures in this field through their change of quality or their own specific properties. Nonetheless, from another point of view, such measures could be categorized into three whole rubrics of dietary, medicinal, and intercourse measures. The next section focuses on those measures specific to turning the fetus to be male.

First, it would be better for both male and female partners to be purged of waste materials, moisture(s), and cold phlegmatic humors. Then, the testes and the ovaries are to be made warm in addition to semen-making organs and the semen itself. Therefore, both male and female partners should be warmer through the means of foodstuff, beverages, and perfumes, enema, and medications that are applied into the vagina, warming ointments, and oils (specifically those which make the ovaries, the uterus, and the semen-producing organs warm). Of course, such manner of warming should be carried out to the extent that the blood would not be spoilt. As regards dietary measures, the following items could receive attention:

- Not drinking too much water. In the case of water drinking, both the wife and the husband should (sort of) sip or drink it gradually because drinking too much water causes the semen to become rare. Excessive drinking of cold water causes the semen to become cold, weakening the fetus.

- Eating strengthening foods with warm quality, including normal Spidbaj (a soup consisting of dill, leeks, onions, peas, cinnamon, pepper, and cumin), meatballs, Ghelye Nargesi (fricassee with Persian carrot), the yolk of soft boiled eggs, lamb meat, rice with milk, and Halva with sugar are effective.

- Consuming ginger jam, parsnip, and bergamot (citron) is recommended.

- Consuming various kinds of meat such as lamb meat (23), ewe, sparrows, quail, salted sea fish, along with spices such as mustard, celery, mint, and pepper are all recommended as well.

- Consuming suitable helpful vegetables such as celery, mint, cress, onion, leek, dried and/or fresh garlic, carrots, parsnips, and eggplant. It is also beneficial to have figs and (ripe) sweet grapes.

- Having snacks (nut-) include pistachios, poplar seeds, red currant, roasted wheat, and peas.

- Cooking meals with warming spices such as marjoram, saffron, bergamot, lemon, Dudaim Melon, amber, musk, mastic, celery seeds, fennel, anise, nettle seeds, caraway, asparagus, cumin, and cinnamon.

- Avoiding certain foods comprising of cold and wet foods, fresh cold water and beverages, pickles and all kinds of sour foods and/or fruits, lentils, curd, broad beans, sumac, sour and/or sour-sweet pomegranates, cucumbers, dried and fresh coriander, lettuce, pumpkin, colostrums, cheese, and camphor. Drunkenness and overeating should be avoided as well.

- Consuming medications taken orally or inhaled or intra-vaginally received attention in ancient medicine literature. Some of these medications included methroditus, Jovaresh [24], ambergris, incense Jovaresh (specialized formulation of highly complex 
nature for gastric disorders), Dva' Al-mosk in winter, Lakh-Lakheh (semi-liquid, inhaled dosage form) with saffron (22), and drinking of animal antidote with doogh.

- Emphasizing the usage of warm-natured perfumes such as musk, saffron, aloeswood, Agarwood possibly combined with "triangle" perfume (Atr-e-Mosalas), and ambergris $(12-14,16)$.

Discussion and Conclusion

The review findings showed that sex selection based on TPM must be considered for the temperament of the sexual organs in a man, the temperament of the sexual organs in a woman, the type of male semen, and timing and manner of sexual intercourse for fetus sex induction purposes. In addition, other intended parameters included the effect of environmental factors, the couple's psychic states during the course of love-making, the couple's age, and measures for male sex induction. In new medicine, various laboratory methods are used for selecting babies' sex before conception. These methods consist of preimplantation genetic diagnosis through which sperms are injected into the ovum through micro-injection, leading to the performance of fertilization after inciting ovulation. It is only after a biopsy from a fetus of 6-8 cells that a blastomere is separated from a fetus of $6-8$ cells. The blastomere's sex is determined through the fluorescence in situ hybridization method or molecular methods. After culturing, the fetus would be transferred into the intended uterus. The mentioned method is of a high level of sensitivity, though it is extremely costly, namely, it is of complex technicalities where the chance of pregnancy turns out to be low (7).

The other method is Microsort in which fluorescentstained sperms are separated through using the flow cytometry apparatus. Then, those sperms containing the intended chromosome are transferred into the uterus by the intrauterine insemination method, which is of wide application among animals (7). However, its application has been well criticized for human beings due to the usage of fluorescent dyes such as Hoechst color and laser rays throughout the Microsort method $(3,8)$.

The next alternative would be the albumin gradient method which comprises of passing on of sperms through human albumin layers so that those sperms containing the $\mathrm{Y}$ chromosome are taken up from those containing $\mathrm{X}$ chromosomes to lie within two differing layers. This method has frequently been applied in sex determination by the mentioned investigators . Some studies have affirmed the above-mentioned method $(3,9)$ while another research reported it as unsuccessful (3).

The next method is the simple modified swim-up which is based upon the motion of sperms and is actually conducted through several stages $(3,10)$. Although the success level of this method has been reported to be arriving at $89 \%$ wanted males as compared to $86 \%$ wanted females, other researchers have not expressed specific opinions about this method (3).

Non-satisfaction in families regarding the sex of the offspring causes great psychic harm to the stability of the family itself. Iranian TPM sages have regarded psychic, mental, somatic, environmental, and the mannerism of sexual intercourse as significant parameters in fetus sex induction. Proper assistance could well potentially be delivered to families if enough attention should be paid to such measures and advice. Treatment interventions and/or dietary measures might be of great assistance in giving families the opportunity to choose children with the desired sex. Although recommendations in the TPM literature have been delivered with some highly peculiar jargon, the vast comprehensive mastery of previous Iranian sages over this issue is demonstrated whereby modern researchers and those interested in more investigation in this field can prospectively propose delving into various facets of such a topic from their own differing standpoints so that a wide variety of dimensions are opened up toward even more comprehensive research and interventional studies in this field.

The review findings can be useful for applying in the practice as complementary medicine, and clinicians can integrate sex selection methods based on TPM in the new medicine.

\section{Authors' Contribution}

SSY, RM and MT designed study, drafted the initial manuscript, collected data, and approved the final manuscript. SB supervised the various stages of study. ZH revised and approved the final manuscript. All authors have confirmed the submitted manuscript and are responding to the study.

\section{Conflict of Interests}

The authors declare that they have no conflict of interests.

\section{Ethical Issues}

The study protocol was approved by the Ethics Committee of Mazandaran University of Medical Sciences, Sari, Iran (IR. MAZUMS.REC.96.10218).

\section{Financial Support}

The study protocol was approved and funded by the Research Committee of Sexual and Reproductive Health Research Center, Mazandaran University of Medical Sciences, Sari, Iran (Code number: 10218).

\section{References}

1. Schulman JD, Karabinus DS. Scientific aspects of preconception gender selection. Reprod Biomed Online. 2005;10 Suppl 1:111-115. doi:10.1016/s1472-6483(10)62217-1

2. Holt WV, O'Brien J, Abaigar T. Applications and interpretation of computer-assisted sperm analyses and sperm sorting methods in assisted breeding and comparative research. Reprod Fertil Dev. 2007;19(6):709-718. doi:10.1071/rd07037

3. Khalili MA, Khani B, Baghazadeh S, Tabibnejad N. Sex selection by using albumin gradient technique for sperm separation in IUI cycles. J Reprod Infertil. 2007;8(3):213-220. [Persian].

4. Cunningham FG, Leveno KJ, Bloom SL, Hauth JC, Gilstrap LC, 
Wenstrom KD. Williams Obstetrics. 22nd ed. McGraw-Hill; 2005.

5. Jacobsen R, Møller H, Engholm G. Fertility rates in Denmark in relation to the sexes of preceding children in the family. Hum Reprod. 1999;14(4):1127-1130. doi:10.1093/ humrep/14.4.1127

6. Polo-Kantola P, Erkkola R, Irjala K, Pullinen S, Virtanen I, Polo O. Effect of short-term transdermal estrogen replacement therapy on sleep: a randomized, double-blind crossover trial in postmenopausal women. Fertil Steril. 1999;71(5):873-880. doi:10.1016/s0015-0282(99)00062-x

7. Missmer SA, Jain T. Preimplantation sex selection demand and preferences among infertility patients in Midwestern United States. J Assist Reprod Genet. 2007;24(10):451-457. doi:10.1007/s10815-007-9157-2

8. Cran DG. $X Y$ sperm separation and use in artificial insemination and other ARTs. Soc Reprod Fertil Suppl. 2007;65:475-491.

9. Pyrzak R. Separation of X- and Y-bearing human spermatozoa using albumin gradients. Hum Reprod. 1994;9(10):17881790. doi:10.1093/oxfordjournals.humrep.a138332

10. Khatamee MA, Horn SR, Weseley A, Farooq $T$, Jaffe SB, Jewelewicz R. A controlled study for gender selection using swim-up separation. Gynecol Obstet Invest. 1999;48(1):7-13. doi:10.1159/000010125

11. Arksey H, O'Malley L. Scoping studies: towards a methodological framework. Int J Soc Res Methodol. 2005;8(1):19-32. doi:10.1080/1364557032000119616

12. ozenbaum H, Chevallier O, Moyal M, Durand G, Perineau $M$, This P. Efficacy and tolerability of pulsed estrogen therapy: a 12-week double-blind placebo-controlled study in highly symptomatic postmenopausal women. Climacteric. 2002;5(3):249-258.

13. Saletu B, Brandstätter N, Metka $M$, et al. Double-blind, placebo-controlled, hormonal, syndromal and EEG mapping studies with transdermal oestradiol therapy in menopausal depression. Psychopharmacology (Berl). 1995;122(4):321329. doi:10.1007/bf02246261

14. Scharf MB, Berkowitz DV, Reape KZ. Effects of synthetic conjugated estrogens A on sleep quality in postmenopausal women with nocturnal diaphoresis and/or hot flushes: a pilot study. Fertil Steril. 2007;88(3):654-656. doi:10.1016/j. fertnstert.2006.11.181

15. Disilva DK. Mojarabate Farangi (Persian). Tehran: Institute of Medical History.

16. Schürmann R, Holler T, Benda N. Estradiol and drospirenone for climacteric symptoms in postmenopausal women: a double-blind, randomized, placebo-controlled study of the safety and efficacy of three dose regimens. Climacteric. 2004;7(2):189-196. doi:10.1080/13697130410001713698

17. Baumgardner SB, Condrea H, Daane TA, et al. Replacement estrogen therapy for menopausal vasomotor flushes. Comparison of quinestrol and conjugated estrogens. Obstet Gynecol. 1978;51(4):445-452. doi:10.1097/00006250197804000-00013

18. Tabari A. Ferdos -Al- Hekmah (Arabic). Tehran: Institute of Medical History; 2012.

19. Shulman LP, Yankov V, Uhl K. Safety and efficacy of a continuous once-a-week 17beta-estradiol/levonorgestrel transdermal system and its effects on vasomotor symptoms and endometrial safety in postmenopausal women: the results of two multicenter, double-blind, randomized, controlled trials. Menopause. 2002;9(3):195-207. doi:10.1097/00042192200205000-00008

20. Simon J, Klaiber E, Wiita B, Bowen A, Yang HM. Differential effects of estrogen-androgen and estrogen-only therapy on vasomotor symptoms, gonadotropin secretion, and endogenous androgen bioavailability in postmenopausal women. Menopause. 1999;6(2):138-146. doi:10.1097/00042192199906020-00011

21. Jorjani SE. Al- Aghraz al- Tibbieh al- Mabahes al- Alaieh 2. Tehran: Tehran University Press; 2005.

22. Simon JA, Bouchard C, Waldbaum A, Utian W, Zborowski J, Snabes MC. Low dose of transdermal estradiol gel for treatment of symptomatic postmenopausal women: a randomized controlled trial. Obstet Gynecol. 2007;109(3):588-596. doi:10.1097/01.aog.0000254160.62588.41

23. Simon JA. Estradiol in micellar nanoparticles: the efficacy and safety of a novel transdermal drug-delivery technology in the management of moderate to severe vasomotor symptoms. Menopause. 2006;13(2):222-231.

Copyright $\odot 2022$ The Author(s); This is an open-access article distributed under the terms of the Creative Commons Attribution License (http://creativecommons.org/licenses/by/4.0), which permits unrestricted use, distribution, and reproduction in any medium, provided the original work is properly cited. 